\title{
Esophageal Neuroendocrine Carcinoma
}

National Cancer Institute

\section{Source}

National Cancer Institute. Esophageal Neuroendocrine Carcinoma. NCI Thesaurus. Code C95619.

An aggressive, high-grade and poorly differentiated carcinoma with neuroendocrine differentiation that arises from the esophagus. The mitotic count is more than 20 per 10 HPF. According to the size of the malignant cells, the prominence of the nucleoli, and the amount of cytoplasm, it is classified either as small or large cell neuroendocrine carcinoma. 